\title{
A Thermodynamic Perturbation Method to Calculate Surface Forces in Confined-Fluid Systems
}

\author{
Sang Woo Han ${ }^{\dagger}$ and Joonkyung Jang

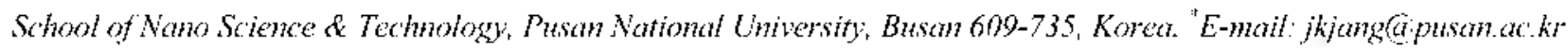 \\ ${ }^{+}$Department of Chemistry and Research Institute of Natural Seien'e, Gyeongsang National Liversity, Jinju 660-701, Korea \\ Received Nowember 12, 2005
}

Key Words : Themodynamic perturbation, Surlace force, Latice gas, Monte Carlo

In studying phase behavior of confined lluids, a latlice gas model' is widely used. It has been successfully applied to the phase behavior of water trapped in a carbon nanotube. 2 We have used a similar model to study the capillary force due to a water meniscus that forms between an $\Lambda F M$ lip and a surface. In the Monte Carlo simulation of a conlined lluid, it is olten reasonable to fix the chemical potential $\mu$ (e.g. in phase ecpuilibria) instead of the number of molecules $N$. It is then sensible to work with a grand-canonical $(\mu V T)$ ensemble. ${ }^{2-4}$ Figure I illustrates a grand-canonical Monte Carlo simulation of a liculid conlined between two surfaces. The licpuid sites, drawn as circles in the figure, mimic water molecules at a relative humidity of $29 \%$. The circular upper surlace is modeled aller a nanoscale $\Lambda F M$ tip, and the llat lower surface corresponds to the surlace probed by the tip. As the inter-swface distance $h$ increases by one latice spacing $l$ [from $\wedge$ to $B$ in Figlare 1], the liquid profile changes (usually, the meniscus narrows in its waist). The force belween two surfaces, $F$, is given by

$$
F(h)=-\left(\frac{\partial \Omega}{\partial h}\right)_{\mu, T}-p\left(\frac{\partial V}{\partial h}\right)_{\mu, T}
$$

, where $\Omega$ and $V$ are the grand potential and volume of the system, respectively, and $p$ is the pressure of the bulk system with a chemical polential $\mu$. The key quantity in the Monte Carlo calculation of equation ( 1 ) is the derivative of $\Omega$ with respect to $h$ (the bulk pressure $p$ can be casily calculated by using an analytic theory such as the density functional theory ${ }^{3}$ ). Within a latice model in general, the derivative is given by the change in $\Omega$ as $h$ increases by $/$. Therefore, in order to calculate the force, one needs $[\Omega(h+h)-\Omega(h)]$, where $\Omega(h+h)$ and $\Omega(h)$ are the grand potentials at intersurface distances of $h+l$ and $h$, respectively.

Unfortunatcly, $\Omega$ is not a direct observable in a standard Monte Carlo simulation. Previously, we used a thermodynamic integration method that ulilizes the following relation,"

$$
\left.\frac{\partial(\beta \Omega)}{\partial \beta}\right|_{\mu, t_{1}}=E-\mu N \quad\left(\beta^{\top}=k_{B} T\right),
$$

where $E$ and $N$ are the energy and number of molecules, respectively. In order to calculate $\Omega_{1}$ we numerically integrated equation (2) as follows. Starting from $\beta=0$ (infinite $T$ where $\Omega$ is known exaclly), we ran simulations for at least 10 intemediate $\beta$ values. $\Lambda t$ each $\beta$ value, we cvaluated the right-hand side of equation (2) by using a standard Montc Carlo method. Obviously, running simulations at intennediale temperatures is the computational bottleneck in the lorce calculation. There is another thermodynamic integration method that involves an integration with respect to $\mu^{3.4}$ but its computational load is nearly identical to that required for the above integration with respecel to $\beta$.

IIere we propose a themodynamic perturbation method to calculate the change in the grand potential. Ist us write the diflerence in the grand potential as

$$
\Omega\left(h+h-\Omega(h)=-k_{h} T \ln [Z(h) / Z(h+h)]\right.
$$

, where $Z(h)$ and $Z(h+l)$ are the partition functions of the systems with inter-surlace distances of $h$ and $h$ । $/$,

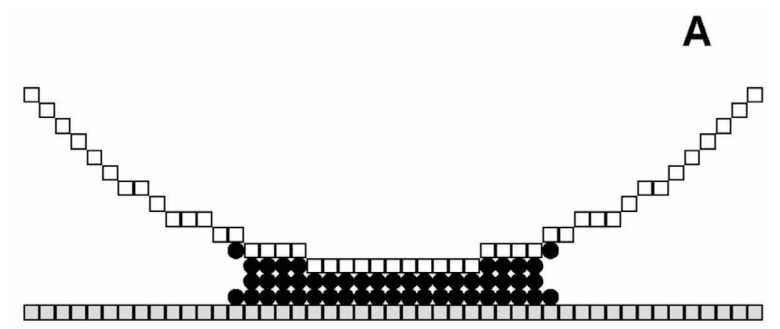

B

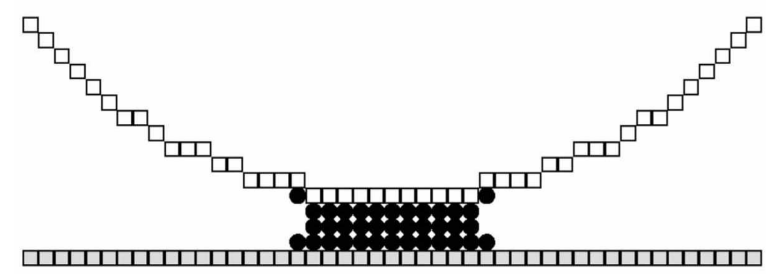

Figure 1. Prolile of a liquid conlined between ino solid surtaces. The upper- and lower- surface siles are draw as open and hilled squares, respecively. The liquid sites (defined as the lattice sites with an average occupancy above $1 / 2$ ) are represented as lilled circles. These ligures represent 2-dimensional cross sections of waler menisci condensed between an $\Lambda F M$ tip and a llat surface under an ambient condition. $A$ lattice gas mode $l^{-4}$ has been used in this simulation. The liquid profile changes when the distance hetween two surlaces, $h$, increases from $3[\Lambda]$ to $4[\mathrm{~B}]$ lallice spacings, /s. 
respectively. The formal expression of $7(h)$ is

$$
Z(h)=\sum_{c_{1}=\{j}^{1} \sum_{c_{2}=\{}^{1} \ldots \sum_{c_{j ;}=0}^{1} \exp \left|-\beta H\left(c_{1}, c_{2}, \ldots c_{B}\right)\right|
$$

, where $B$ is the total number of lattice sites, and $c,(l=1,2$, $\cdots, B$ ) is the occupation number (0 or I) of the ith site. The effective energy $H$ in equation (4) is defined as

$$
H\left(c_{1}, c_{2}, \ldots c_{B}\right)=E\left(c_{1}, c_{2}, \ldots c_{B}\right)-\mu N\left(c_{1}, c_{2}, \ldots c_{B}\right)
$$

, where we wrote the energy $E\left(c_{1}, c_{2}, \ldots c_{\beta}\right)$ and number $N\left(c_{1}, c_{2}, \ldots c_{\beta}\right)$ as finctions of the occupation number of cach site. $E\left(c_{1}, c_{2}, \ldots c_{B}\right)$ is typically given as a sum of intermolecular and molecule-surlace interactions both taken to be of a nearest-neighbor type. Now suppose the total number of lattice sites at an inter-surface distance of $h+/$ is $B^{\prime}(>B)$. Note

$$
\begin{aligned}
Z(h) & =2^{\left(B-F^{\prime}\right)} \\
& \times \sum_{c_{1}=0}^{\vdots} \sum_{c_{2}=0}^{1} \cdots \sum_{C_{A}=0}^{1} \cdots \sum_{c_{n}=0}^{1} \exp \left|-\beta H\left(c_{1}, c_{2}, \ldots c_{B}\right)\right| .
\end{aligned}
$$

Using the above equation, we can write the ratio of partition functions in equation (3) as

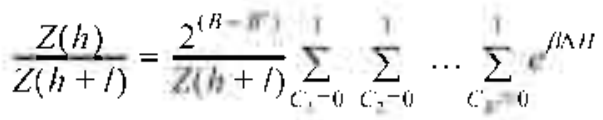

$$
\begin{aligned}
& \times \exp \left[-\beta H\left(c_{1}, c_{2}, \ldots c_{B^{\prime}}\right) \mid=2^{(B-B)}\left\langle e^{\beta \Delta H}\right\rangle_{H, h 1, T}\right.
\end{aligned}
$$

, where $\Delta I I=I /\left(c_{1}, c_{2}, \ldots c_{B}, \ldots, c_{B^{\prime}}\right)-I I\left(c_{1}, c_{2}, \ldots c_{B}\right)$, and $(\ldots)_{\mu, H \cdot T, T}$ means the ensemble average for a fixed chemical polential $\mu$, an inter-surface distance $h+l$, and temperature $T$. Using equation (7), we can write equation (3) as

$$
\begin{aligned}
& \Omega(h+h)-\Omega(h)= \\
& \quad k_{B} T\left|\left(B-B^{\prime}\right) \ln 2+\ln \left(\left\langle\exp \left|\Delta I / / k_{B} T\right|\right\rangle_{(B, i-1, T}\right)\right| .
\end{aligned}
$$

The above equation expresses the diflerence in the grand potential as an ensemble average of exponentiated $\Delta / /$.

In principle, equation (8) can be evaluated by running a simulation at a single themodynamic state. $A n$ accurate evaluation of equation (8) however will require sampling a range of $\Delta H$ much broader than obtained in a standard Monte Carlo method. There are advaneed Monte Carlo technigues designed to meet such a requirement. Overlapping distribution method and umbrella sampling ${ }^{7}$ would be examples. Another numerical difficulty arises when typical $\Delta / /$ values are large, causing a divergence in $\left\langle\exp \left[\Delta H / k_{R} T\right]\right\rangle_{t i, i, i, T}$. To make $\Delta H$ smaller in magnitude, one could introduce an intermediate geometry as follows. Let us consider the case where the inter-surface distance $h$

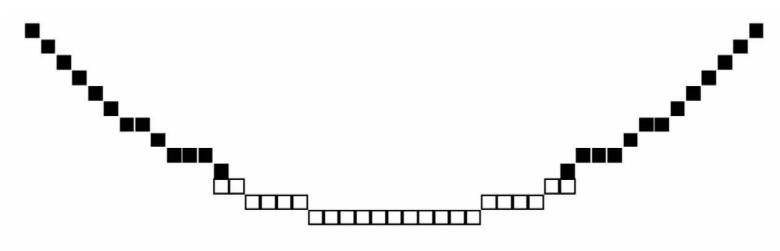

שחדזחשתח

Figure 2. No indismediate geometry of Figure $I \wedge(b-3 /)$ and Figure I 3 (kfth. $A$ s in Figure 1. gray-filled squares represent the lower surtace sitas. Open and black-filled squares correspond to the tip-surlace sites al $h=4 /$ and $h=3 /$, respectively. The liquid prolite for lis geometry (not shown) is expected to be in the middle of the two profïles shown in Figure I.

varies from $3 /$ to $4 /$ (as in Figure 1). One can think of this transition in the geometry as a two-step process by inserting an intermediate geometry shown in Figure 2. In this choice of an intermediate geometry (one can think of various other geometries dilferent from Figure 2), the hall of the upper surface sites are identical to the upper surface sites at $h=37$ (Figure $1 \mathrm{~A}$ ), and the rest of the upper surface sites corresponds to the upper surface sites at $h=4 /$ (Figure $1 \mathrm{~B}$ ). The transition from Figure $1 A$ to Figure $1 \mathrm{~B}$ can be divided into the transitions from Figure $1 \wedge$ to Figare 2 and from Figure 2 to Figure IB. Fyuation (8) can be applicd to each slep, and the net change in $\Omega$ is given by the sum of $\Omega$ change in each step. In this way, one can vary the system geometry gradually, and $\Delta H$ values in simulation will be smaller. The decrease in the magnitude of $\Delta / /$ will be helpful in preventing the divergence of exponential function in equation (8). This scheme, combined with the advaneed Monte Carlo techniques, is expected to be less time-consuming than thermodynamic integration methods (which typically require 10 additional simulations for a given thermodynamic state). In summary, cquation (8) will provide an efficient computational method compared to the previous Monte Carlo methods using a thermodynamic integration.

Acknowledgement. IJ gratefully acknowledges tho linancial support from Korean Rescarch Foundation Grant No. R05-2004-000-10484-0.

\section{References}

I. Itill, T. I. Statisticat Mechanics; McGraw-Itill: Now York. 1956; (hap. 7.

2. Maibaum, I., Clandler, D. J. Phis. Chem. B 2003, 107, 1189.

3. Jang. J.; Schats, G. C.; Ratner; M. A. J. Chem. Phrs. 2004, /2t, 157.

4. Jang. J.; Schatz, G. C., Ratner, M. A. Phis. Rev Lett. 2003, 90, 150104

5. Livans, R.; Marini Buttolo Marconi, U. J. Chem. Phis. 1987, 86, 7138.

6. Peterson, B. K.: Cubbins, K. I. Mol. Phn's. 1987, 62, 215.

7. Frenkel, D.: Smit, B. Unterstunding Holecular Simutation; Academic Press: San Dicgo, 2002; Chap. 7. 